\title{
Combination of proton pump inhibitor and rebamipide, a free radical scavenger, promotes artificial ulcer healing after endoscopic submucosal dissection with dissection size $>40 \mathrm{~mm}$
}

\author{
Hiroshi Araki, ${ }^{1, *}$ Tomohiro Kato, ${ }^{1,2}$ Fumito Onogi, ${ }^{1}$ Takashi Ibuka, ${ }^{1}$ Akihiko Sugiyama, ${ }^{2}$ Takayuki Nakanishi, ${ }^{1}$ \\ Tomohiko Sugiyama, ${ }^{1}$ Eiichi Tomita ${ }^{2}$ and Hisataka Moriwaki ${ }^{1}$
}

1Department of Gastroenterology, Gifu University Hospital, Gifu 501-1194, Japan

${ }^{2}$ Department of Gastroenterology, Gifu Municipal Hospital, Gifu 503-8502, Japan

In our previous study, the healing effect of proton pump inhibitor plus rebamipide for endoscopic submucosal dissection-related artificial ulcer smaller than $\mathbf{4 0} \mathbf{~ m m}$ showed statistical significance. However, such effect of the combination was not yet clear for ulcers with dissected diameter more than $\mathbf{4 0} \mathbf{~ m m}$. The aim of this present study was to resolve this problem under sufficient statistical power, with adequate sample size. We conducted a randomized controlled study. Either the proton pump inhibitor mono-therapy or the combination therapy was prescribed for 28 days after endoscopic submucosal dissection. Eighty-seven patients were eligible for outcome evaluation. Combination therapy was significantly superior to mono-therapy, $27.8 \%$ vs $0 \%$ reached healing stage (scar stage) in cases with ulcers of dissection diameter more than $40 \mathrm{~mm}$. In conclusion, the combination therapy with rebamipide was favorable regimen in patients with larger artificial ulcer after endoscopic submucosal dissection.

Key Words: endoscopic submucosal dissection (ESD), proton pump inhibitor (PPI), rebamipide, artificial ulcer, ulcer healing

E ndoscopic submucosal dissection (ESD) has become popular around the world to treat patients with early gastric cancer. The current interest in ESD is regarding its application for larger tumor size. As the problematic issue in ESD procedure, delayed bleeding after ESD from artificial ulcer was focused. Gotoda et al..$^{(1)}$ reported that frequency was up to $7 \%$. Moreover, Uedo et al. ${ }^{(2)}$ demonstrated that the size of a tumor larger than $20 \mathrm{~mm}$ was an independent predictive factor for delayed bleeding. Okada et $a l^{(3)}$ demonstrated that a dissected specimen of diameter greater than $40 \mathrm{~mm}$ was the only significant factor associated with delayed bleeding. Expand application of ESD lead to increase the risk of delayed bleeding from artificial ulcer. Moreover, bleeding in patients discharge is fatal. Therefore, management of larger size artificial ulcer is important. Although proton pump inhibitor (PPI) or $\mathrm{H}_{2}$ receptor antagonist $\left(\mathrm{H}_{2} \mathrm{RA}\right)$ is effective for the prevention of bleeding and the healing of artificial ulcer after ESD, ${ }^{(2,4)}$ it has a therapeutic limitation. In our previous study, the healing ratio in 4 weeks PPI mono-therapy was decreasing as larger as dissection specimen size: $33.3 \%(17 / 51)$ for specimen size $<40 \mathrm{~mm}$ and $20.0 \%(2 / 10)$ for $\geq 40 \mathrm{~mm} .{ }^{(5)}$ Administration of rebamipide, a cytoprotective agent, with PPI showed significantly superior healing effect on ulcers with larger dissected size of 20 to $40 \mathrm{~mm}$ as compared with PPI mono-therapy. Such effect of the combination was suggested also for ulcers with a diameter greater than $40 \mathrm{~mm}: 75 \%(6 / 8)$ by the combination therapy and $20 \%(2 / 10)$ by the mono-therapy. However, there was no statistical significance ( $p=0.058$ by Fischer's exact test) because of less statistical power due to the small number of patients with such dissection size. Therefore, we conducted the following study to prove the healing effect of the combination therapy for artificial ulcer with a diameter greater than $40 \mathrm{~mm}$ with adequate sample size calculated from our previous data.

\section{Methods}

Subjects. This study was conducted from 2007 to 2010 at Gifu University Hospital Endoscopy Center and Gifu Municipal Hospital. The study protocol was approved by the Institutional Review Board for Human Research at each hospital. Inclusion criteria were all patients with gastric adenoma or early cancer who attended our hospitals for the purpose of ESD with a predicted resection size over $20 \mathrm{~mm}$. Exclusion criteria were those with repeat bloody vomitus, major organ failure such as cardiac and pulmonary complications, or drug allergy. Those receiving concomitant administration of anticoagulants and anti-inflammatory drugs were also excluded. Written informed consent was obtained from all participants.

Study drugs and treatment schedule. Patients were admitted one day before ESD and hospitalized for at least 7 days post-ESD. All patients received an intravenous administration of omeprazole on the first two days after ESD. Participants then took either PPI (omeprazole $20 \mathrm{mg} /$ day, lansoprazole $30 \mathrm{mg} /$ day or rabeprazole $10 \mathrm{mg} /$ day) alone or PPI plus $100 \mathrm{mg}$ rebamipide 3 times/day for the following 26 days.

Endpoints. The primary endpoint was to evaluate the artificial ulcer healing ratio of the combination therapy compared with the PPI mono-therapy in all patients after ESD. The secondary endpoint was to evaluate the effect of the combination therapy in patients with a dissection size greater than $40 \mathrm{~mm}$.

Outcome evaluation. Artificial ulcer healing was evaluated

*To whom correspondence should be addressed.

E-mail: araara@gifu-u.ac.jp 


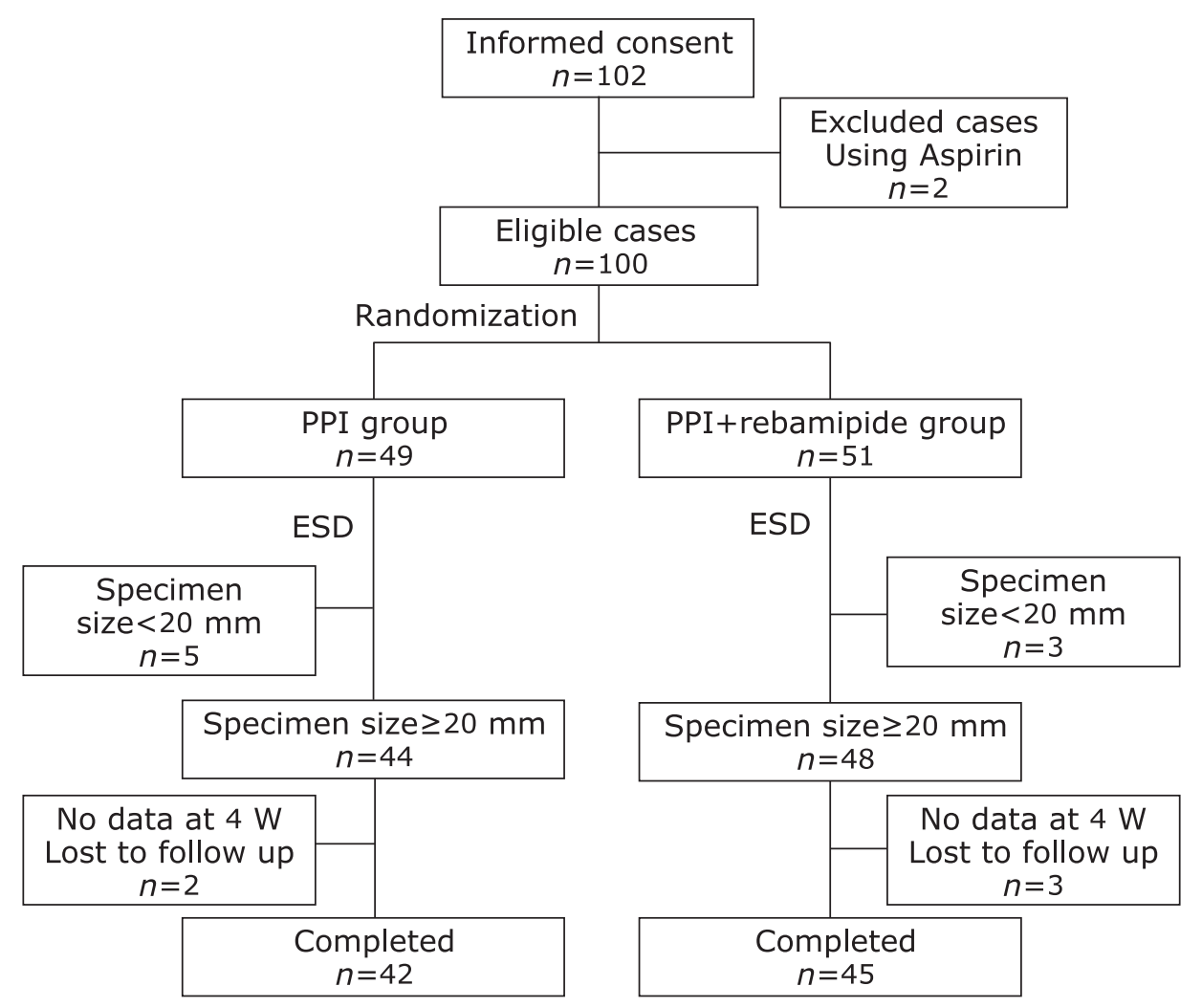

Fig. 1. Patient flow.

by endoscopy on the 28th day. The Sakita and Miwa classification, the scar stage was defined as healing of ulcer, was an evaluation scale for the ulcer stage. ${ }^{(5,6)}$ The dissection size was measured by pinning the specimen flat on a rubber plate. Endoscopic evaluation for the ulcer stage on the 28th day was judged by a representative blinded gastroenterologist, H.A. in Gifu University Hospital.

Calculation of the sample size. In our previous study on ESD ulcers $\geq 20 \mathrm{~mm},{ }^{(5)} 68 \%$ of patients in the combination group progressed to scar stage while only $35 \%$ progressed in the PPI mono-therapy group (33\% improvement, $p=0.011)$. Therefore, at least a total of 35 cases were calculated as required sample size in one arm with $\alpha$-error $<0.05$ and $\beta$-error $<0.2$. Regarding cases with ulcer greater than $40 \mathrm{~mm}, 6$ of $8(75 \%)$ patients in the combination group progressed to scar stage as compared with 2 of $10(20 \%)$ in the PPI mono-therapy group. These data indicate that at least 12 cases with such ulcer size were needed in one arm. In addition, only $30 \%$ of all patients had this artificial ulcer size in the previous study. Thus, the required number of total cases in one arm was 40 . By adding $10 \%$ possible ineligibility in ulcer size and $10 \%$ dropouts, 48 was set as the target sample size in one arm.

Statistical analysis. Baseline demographic and clinicopathologic data were compared using the chi-square test or MannWhitney $U$ test. We used the chi-square test or Fisher's exact test with a $95 \%$ confidence interval to compare the endpoint ulcer stage between the two groups. The significance of difference was declared with a $p$ value of less than 0.05 . All statistical analyses were performed using JMP ver. 8.0.2 software (SAS Institute, Cary, NC).

\section{Results}

Patient flow. As shown in Fig. 1, a total of 100 patients were eligible and randomly assigned to the two groups. ESD was successfully performed and was judged as curative histopathologically in all cases. In 3 patients in the combination therapy group and in 5 cases in the PPI mono-therapy group, the dissected specimen size did not reach $20 \mathrm{~mm}$. Lost to follow-up were 3 in the combination group, and 2 in the mono-therapy group.

Baseline demographic and clinicopathologic data. The baseline demographic and clinicopathologic data were not different between the 2 groups, as shown in Table 1 .

The effect of combination therapy. The ratio of S1 stage at 28 days after ESD was 6 of $42(14.3 \%)$ in the mono-therapy group, and 20 of $45(44.4 \%)$ in the combination group, respectively (absolute difference: $30.1,95 \%$ CI: $12.1-48.1)(p=0.0021)$ (Fig. 2). In cases with dissection diameter greater than $40 \mathrm{~mm}$, the rate of $\mathrm{S} 1$ stage was 0 of $16(0 \%)$ in the mono-therapy group, and 5 of $18(27.8 \%)$ in the combination group (absolute difference: $27.8,95 \%$ CI: 7.0-48.4) ( $p=0.0465)$ (Fig. 2). For ulcers between 20 and $40 \mathrm{~mm}$, the rates were $23.0 \%(6 / 26)$ and $55.6 \%(15 / 27)$, respectively (absolute difference: 32.6, 95\% CI: 7.7-57.2) $(p=0.0244)$ (Fig. 2).

\section{Discussion}

In our previous data, the combination therapy was useful as compared with PPI mono-therapy for ESD-ulcer healing. However, the effect of the combination was not significant on ulcers larger than $40 \mathrm{~mm},{ }^{(5)}$ because the statistical power was insufficient due to the small sample size of this ulcer size. In order to address this concern, we analyzed the proportion of patients with larger ulcers in the total ESD cases referring to our previous data, and designed this study with 48 cases as the target for one arm. As a result, healing ratio of artificial ulcer rose in the combination therapy group by an absolute difference of $30.1 \%$ (95\% CI: $12.1-48.1)$ in all cases. Moreover, the ratio was also improved by a similar 
Table 1. Baseline demographic and clinicopathologic data of PPI alone and PPI + rebamipide combination groups

\begin{tabular}{|c|c|c|c|c|}
\hline & & $\begin{array}{c}\text { PPI } \\
(n=42)\end{array}$ & $\begin{array}{c}\mathrm{PPI}+\underset{(n=45)}{\text { rebamipide }} \\
(n=45\end{array}$ & \\
\hline Age (years, median (range)) & & $69.5(52-85)$ & $71(45-87)$ & ns \\
\hline Gender (male/female) & & $30 / 12$ & $30 / 15$ & ns \\
\hline \multirow[t]{3}{*}{ Histopathology } & Carcinoma & 32 & 36 & \multirow{3}{*}{ ns } \\
\hline & Adenoma & 9 & 9 & \\
\hline & Cartinoid & 1 & 0 & \\
\hline \multirow[t]{4}{*}{ Localization of gastric tumor } & Cardia & 1 & 2 & \multirow{4}{*}{$n$} \\
\hline & Body & 20 & 17 & \\
\hline & Angle & 8 & 11 & \\
\hline & Antrum & 13 & 15 & \\
\hline Tumor size (mm, median (range)) & & $18.5(4-68)$ & $18(2-73)$ & ns \\
\hline Specimen size ( $\mathrm{mm}^{2}$, median (range)) & & $1130(396-6059)$ & $1024(425-5740)$ & ns \\
\hline
\end{tabular}

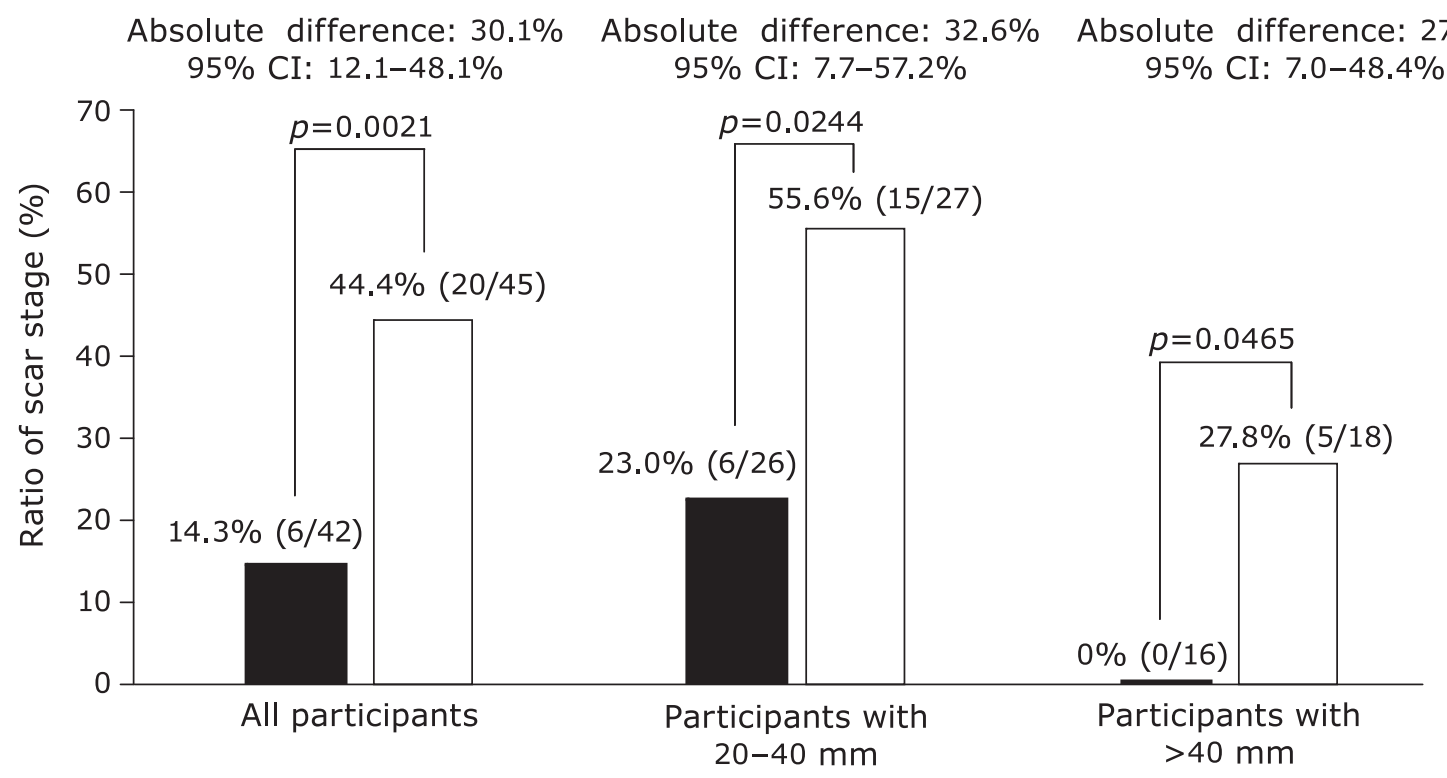

Fig. 2. The ratio of Scar stage at 28 days after ESD. Cl: confidential interval, chi-square test.

absolute difference of $27.8 \%(95 \%$ CI: $7.0-48.4)$ in ulcers larger than $40 \mathrm{~mm}$.

Although PPI is the standard of care for ESD-related artificial ulcer and bleeding after ESD, Fujiwara et al. ${ }^{(7)}$ reported that PPIs and acid-suppressive agents alone have a limited effect in low acid secretion, such as severe atrophic condition in the gastric mucosa. Terano et $a l^{\left({ }^{(8)}\right.}$ reported that 7 weeks of rebamipide treatment without acid-suppressive agents promoted gastric ulcer healing after eradication therapy compared with a placebo treatment. Further evidence of the combination therapy for ESD ulcer has recently been added by Fujiwara et al. ${ }^{(7)}$

Rebamipide promote healing of mucosal injury by inducing the up-regulation of EGF and EGF receptors, ${ }^{(9)}$ and by phosphorylation of extracellular signal-regulated kinase (ERK) and activation of Rho kinase. ${ }^{(10)}$ Moreover, rebamipide maintained gastric mucosal integrity by increasing prostaglandin concentration. ${ }^{(11)}$

\section{References}

1 Gotoda T. Endoscopic resection of early gastric cancer. Gastric Cancer 2007; 10: 1-11.
Patients taking rebamipide concomitantly with PPI or $\mathrm{H}_{2} \mathrm{RA}$ had mucosal injury less frequently than those taking acid suppressants plus other mucoprotective drugs. ${ }^{(12)}$ Our study design employed 4 weeks of treatment after ESD according to the report by Uedo et al. ${ }^{(2)}$ that most bleeding after ESD occurred within 28 days. Therapeutic strategy for artificial ulcer healing within 4 weeks may thus have a clinical significance to prevent bleeding after ESD. Major bleeding in both vomiting and stool was not actually observed in this study.

In conclusion, combination therapy for artificial ulcer healing within 4 weeks might be useful to prevent ESD-related complications in patients with larger dissection area.

\section{Conflict of Interest}

No potential conflicts of interest were disclosed. 
scopic submucosal dissection of early gastric cancer: a prospective randomized controlled trial. Am J Gastroenterol 2007; 102: 1610-1616.

3 Okada K, Yamamoto Y, Kasuga A, et al. Risk factors for delayed bleeding after endoscopic submucosal dissection for gastric neoplasm. Surg Endosc 2011; 25: 98-107.

4 Takeuchi N, Umegaki E, Takeuchi T, Murano M, Yoda Y, Tokioka S, Higuchi K. Gastric ulcer healing after treatment of endoscopic submucosal dissection in Japanese: comparison of $\mathrm{H}_{2}$ receptor antagonist and proton pump inhibitor administration. J Clin Biochem Nutr 2011; 49: 216-221.

5 Kato T, Araki H, Onogi F, et al. Clinical trial: rebamipide promotes gastric ulcer healing by proton pump inhibitor after endoscopic submucosal dissection - a randomized controlled study. J Gastroenterol 2010; 45: 285-290.

6 Sakita T, Fukushima H. Endoscopic diagnosis. In: Yoshitoshi Y, ed. Ulcer of the Stomach and Duodenum (in Japanese). Tokyo: Nankodo, 1971; 198-208.

7 Fujiwara S, Morita Y, Toyonaga T, et al. A randomized controlled trial of rebamipide plus rabeprazole for the healing of artificial ulcers after endoscopic submucosal dissection. J Gastroenterol 2011; 46: 595-602.
8 Terano A, Arakawa T, Sugiyama T, et al. Rebamipide, a gastro-protective and anti-inflammatory drug, promotes gastric ulcer healing following eradication therapy for Helicobacter pylori in Japanese population: a randomized, double-blind, placebo-controlled trial. J Gastroenterol 2007; 42: 690-693.

9 Tarnawski AS, Chai J, Pai R, Chiou SK. Rebamipide activates genes encoding angiogenic growth factors and $\operatorname{Cox} 2$ and stimulates angiogenesis: a key to its ulcer healing action? Dig Dis Sci 2004; 49: 202-209.

10 Takagi T, Naito Y, Uchiyama K, et al. Rebamipide promotes healing of colonic ulceration through enhanced epithelial restitution. World J Gastroenterol 2011; 17: 3802-3809.

11 Tanigawa T, Watanabe T, Ohkawa F, et al. Rebamipide, a mucoprotective drug, inhibits NSAIDs-induced gastric mucosal injury: possible involvement of the downregulation of 15-hydroxyprostaglandin dehydrogenase. $J$ Clin Biochem Nutr 2011; 48: 149-153.

12 Yamamoto T, Isono A, Mishina Y, et al. Gastroduodenal mucosal injury in patients taking low-dose aspirin and the role of gastric mucoprotective drugs: possible effect of rebamipide. J Clin Biochem Nutr 2010; 47: 27-31. 